\title{
A new inflammatory marker in overweight patients: triggering receptor expressed on myeloid cells-1
}

\author{
Cifci $\mathrm{A}^{1}$, Durmaz Ceylan $\mathrm{S}^{2}$, Oklu K ${ }^{1}$, Gungunes $\mathrm{A}^{2}$, Katar $\mathrm{D}^{3}$, Karahan $\mathrm{I}^{1}$, Boyunaga $\mathrm{H}^{4}$, \\ Dindar Badem $\mathrm{N}^{4}$
}

Kirikkale University, School of Medicine, Department of Internal Medicine, Kirikkale, Turkey. dr.aydin.71@hotmail.com

\begin{abstract}
BACKGROUND: Triggering receptor expressed on myeloid cells 1 (TREM-1) is secreted by phagocytes in adipose tissue and it also upregulates the expression of genes involved in the inflammatory response and atherosclerotic conditions. This study was aimed to investigate the serum TREM-1 levels in overweight patients.

METHODS: Twenty-eight subjects in the overweight group (OG) and 20 age-matched healthy subjects in the control group (CG) (BMI $27.6 \pm 1.2$ vs $23.1 \pm 2.17 \mathrm{~kg} / \mathrm{m}^{2}$, respectively, $p<0.001$ ) were included in the study. The serum STREM-1 level was measured by ELISA. The homeostasis model assessment score (HOMA-IR) was also calculated.

RESULTS: The mean TREM-1 levels were significantly higher in OG than in CG $(407.3 \pm 323.7$ vs

$150.3 \pm 152.7 \mathrm{pg} / \mathrm{mL}$, respectively, $\mathrm{p}<0.001)$. The HOMA-IR score was also significantly higher in OG than in CG $(3.42 \pm 3.63$ vs $2.77 \pm 1.61$, respectively). A positive correlation was detected between TREM-1 and BMI $(r=0.318, p=0.028)$.

CONCLUSIONS: This study mainly demonstrated that a high serum TREM-1 level might be an early inflammatory marker in overweight patients. We found that TREM-1 might be associated with BMI in overweight patients regardless of insulin resistance (Tab. 1, Ref. 21). Text in PDF www.elis.sk.

KEY WORDS: TREM-1, insulin resistance, overweight subjects.
\end{abstract}

\section{Introduction}

Adipose tissue macrophages are responsible for a low-grade inflammation that causes metabolic dysfunction and insulin resistance in obese patients (1). The adipose tissue secretes different cytokines in obese and non-obese people. Non-obese subjects mainly have M2 macrophages and their adipose tissue secretes anti-inflammatory cytokines such as: IL-4, IL-10, and IL-13. However, obese subjects show a predominance of M1 macrophages, CD4+T lymphocytes, CD8+ T lymphocytes, B lymphocytes, and mast cells. Thus, the proinflammatory profile indicated by the high IFN- $\gamma$, TNF- $\alpha$, MCP-1, IL- 6 , IL- $1 \beta$ levels and the developing adipocyte necrosis release free fatty acids into the circulation in obese subjects. Increased secretion of the chemokine MCP-1 by adipocytes is responsible for macrophage accumulation in adipose tissues, inducing insulin resistance in addition to a low-grade in-

${ }^{1}$ Kirikkale University, School of Medicine, Department of Internal Medicine, Kirikkale, Turkey, ${ }^{2}$ Kirikkale University, Department of Endocrinology, Kirikkale, Turkey, ${ }^{3}$ Yenimahalle Training and Research Hospital, Department of Chest Diseases, Ankara, Turkey, and ${ }^{4}$ Kirikkale University, School of Medicine, Department of Biochemistry, Kirikkale, Turkey

Address for cprrespondence: A. Cifci, Kirikkale University, School of Medicine, Department of Internal Medicine, Yahsihan, 71450 Kirikkale, Turkey.

Phone: +905319291705 flammation(2). Another driving factor in inflammations associated with obesity is the activation of Toll-like receptor (TLR), especially of TLR-2 and TLR-4. TLRs are exclusive to the innate immune system and are generally activated by pathogen-associated molecular patterns such as LPS. Further, they induce inflammation via activation of the NF- $\mathrm{KB}$ pathway (3). The triggering receptors expressed on myeloid cells 1 (TREM-1) and toll-like receptors (TLRs) are believed to interact synergistically to promote inflammation (4).

TREM-1 is secreted by monocytes/macrophages and neutrophils in the adipose tissue. TREM-1 is from the immunoglobulin superfamily and is a $30 \mathrm{kDa}$ glycoprotein that activates several proinflammatory chemokines such as: MCP-1, MCP-3, MIP-1alfa, IL-8 and cytokines such as TNF-alfa and CD86 $(5,6)$. TREM-1 activates the Janus kinase 2 (JAK2), protein kinase B (PKB/ AKT) and extracellular signal-related kinase (ERK1/2) pathways to upregulate the expression of genes involved in the inflammatory response (7). It is also widely acknowledged that an increase in TREM-1 levels increases insulin resistance in morbidly obese and diabetic patients (8). However, there is no other study on the TREM-1 levels of overweight individuals. Becoming overweight may be a risk for metabolic disorders and may be burden on healthcare systems (9).

This study was aimed to investigate the relationship between serum TREM-1 levels and insulin resistance parameters in overweight patients. 


\section{Material and methods}

Our study was conducted on female patients, who presented to Kirikkale University School of Medicine's Internal Medicine and Endocrinology Outpatient Clinics. A total of 28 overweight women (28.27 \pm 5.88 years old; BMI: $25-29.9 \mathrm{~kg} / \mathrm{m}^{2}$ ) and 20 age-matched women with normal weight (26.10 \pm 7.09 years old; BMI: 18-24.9 $\mathrm{kg} / \mathrm{m}^{2}$ ), who volunteered, were included in the study.

Exclusion criteria were as followed: History of drugs such as: oral contraceptive agents, antioxidant agents, or anti-thrombocyte agents; endocrine disease (e.g. hypothyroidism, hypogonadism, polycystic ovary syndrome, Cushing syndrome); multisystemic disease (e.g. diabetes mellitus, hypertension, coronary artery disease, chronic obstructive pulmonary disease, asthma); hematologic conditions (e.g. anemia, thrombocytopenia); history of thrombosis and thrombophilia; infectious disease; alcohol abuse or smoking; male subjects; and refusal to participate in the study.

All participants provided a signed informed consent. Approval for the present study was obtained from the Institutional Review Board.

\section{Anthropometric evaluation}

Bioelectrical impedance analysis was performed with the "Tanita-BC 420 MA" device. These subjects were told not to eat anything at least 4 hours prior to the measurement, not to drink anything including caffeine-containing drinks, not to enter the sauna or bathroom, not to drink alcohol until drawing samples 24 hours before. The data obtained by bioelectrical impedance measurements such as: height $(\mathrm{cm})$, weight $(\mathrm{kg})$, body mass index $\left(\mathrm{kg} / \mathrm{m}^{2}\right)$, fat mass $(\mathrm{kg})$, fat percentage $(\%)$, muscle mass $(\mathrm{kg})$, bone mass $(\mathrm{kg})$ were analyzed.

All blood samples were drawn from the antecubital vein between 8 a.m. and 9 a.m. after a fasting period of 8 hours. Blood samples were centrifuged at $3500 \mathrm{rpm}$ for 10 minutes and their sera were separated. The sera were then divided into groups and stored at $-80^{\circ} \mathrm{C}$ until the analysis. All sera were thawed at room temperature on that day. Serum human TREM-1levels were measured by the ELISA method using the HUMAN TREM-1 kit (Cata$\log$ No: CSB-E04836h) from Cusabio. The results were defined in $\mathrm{pg} / \mathrm{ml}$ (detection range 31.25-2000 pg/ml, minimum measurable dose $7.8 \mathrm{pg} / \mathrm{ml}$; intraassay precision $\mathrm{CV}<8 \%$, interassay precision $\mathrm{CV}<10 \%$ ).

\section{Measurement of insulin resistance}

The HOMA-IR score was measured with the following formula after measuring the fasting plasma glucose and insulin: HOMA-IR=fasting plasma glucose $(\mathrm{mmol} / \mathrm{L}) \times$ fasting plasma insulin $(\mathrm{mU} / \mathrm{mL}) / 22.5$

\section{Other hormonal and biochemical analyses}

Fasting plasma glucose and insulin levels were measured using cobas ${ }^{\circledR}$ e 601 brand original Roche diagnostic kits (Roche Diagnostics Gmbh, Sandhofer Strasse 116, D-68305 Mannheim). Friedewald's Formula (LDL=Total cholesterol-(VLDL + HDL)) was used to calculate LDL-cholesterol levels for triglyceride levels below $400 \mathrm{mg} / \mathrm{dl}$. The Roche Diagnostics Cobas c501 autoanalyzer was used for CRP levels.

\section{Statistical analysis}

For all statistical analysis, IBM SPSS 25 was used. The Shapiro-Wilk test was used for evaluating a normal distribution. The mean and standard deviation was given for normally distributed values, median (minimum-maximum) were used for not normally distributed. The Mann-Whitney-U test or Student $t$ test were used for two group comparisons. The Spearman's correlation analysis was used for describing relationship between two values. $\mathrm{p}<0.05$ was accepted as significant.

\section{Results}

The bioelectrical impedance analysis data obtained using the TANITA device were as followed in the overweight group and the control group, respectively: body weight $71.5 \pm 8.1 \mathrm{~kg}$ vs $58.1 \pm 7.6 \mathrm{~kg}(\mathrm{p}<0.001)$, fat percentage $36.9 \pm 14.0 \% \mathrm{vs} 23.6 \pm 7.2 \%$ $(\mathrm{p}<0.001)$, and fat mass $25.7 \pm 6.2 \mathrm{~kg}$ vs $14.1 \pm 5.6 \mathrm{~kg}(\mathrm{p}<0.001)$. Other anthropometric characteristics of the study groups are shown in the Table 1.

Serum TREM-1 levels were significantly higher in overweight female subjects compared to the control group (254.6 \pm 29.2 vs $43.8 \pm 14.0 \mathrm{pg} / \mathrm{ml}$ respectively; $<<0.001$ ) (Tab. 1). There was no significant difference in serum lipid parameters or serum CRP levels, when compared to the control subjects. These results are presented in the Table 1. The Spearman's correlation analysis revealed a positive correlation between the serum TREM-1 levels and BMI ( $\mathrm{r}=0.318$, $\mathrm{p}=0.028)$. Serum TREM-1 levels were not significantly correlated with other metabolic parameters.

\section{Discussion}

Based on the findings of this study, serum TREM-1 levels were higher in overweight female participants than in the control subjects. In addition, the increase in TREM-1 levels in overweight women seemed to be independent of the fat mass and insulin re-

Tab. 1. Anthropometric characteristics of the study groups.

\begin{tabular}{lccc}
\hline & Study group & Control group & $\mathrm{p}$ \\
\hline Age, years & $30.0 \pm 9.2$ & $26.7 \pm 7.4$ & 0.226 \\
BMI, $\mathrm{kg} / \mathrm{m}^{2}$ & $27.2 \pm 1.3$ & $21.9 \pm 2.2$ & $<0.001$ \\
Height, $\mathrm{cm}$ & $161.7 \pm 7.9$ & $163.0 \pm 8.4$ & 0.75 \\
Weight, $\mathrm{kg}$ & $71.5 \pm 8.1$ & $58.1 \pm 7.6$ & $<0.001$ \\
Fat \% & $36.9 \pm 14.0$ & $23.6 \pm 7.2$ & $<0.001$ \\
Fat mass, kg & $25.7 \pm 6.2$ & $14.4 \pm 5.6$ & $<0.001$ \\
Muscle mass, kg & $41.5 \pm 3.0$ & $44.9 \pm 4.0$ & 0.004 \\
Bone mass, kg & $2.2 \pm 0,1$ & $2.4 \pm 0.2$ & 0.006 \\
TREM-1 pg/ml & $254.6 \pm 292.0$ & $43.8 \pm 14.0$ & $<0.001$ \\
Fasting plasma glucose, mg/dl & $90.8 \pm 10.2$ & $86.4 \pm 7.0$ & 0.1 \\
HOMA-IR score & $3.2 \pm 3.4$ & $1.9 \pm 1.6$ & 0.003 \\
Total cholesterol, mg/dl & $177.3 \pm 28.8$ & $171.4 \pm 26.2$ & 0.52 \\
LDL cholesterol, mg/dl & $105.9 \pm 24.4$ & $99.2 \pm 26.2$ & 0.06 \\
HDL cholesterol, mg/dl & $52.9 \pm 9.6$ & $61.6 \pm 17.6$ & 0.09 \\
Triglyceride, mg/dl & $91.4 \pm 24,7$ & $99.5 \pm 47.5$ & 0.9 \\
CRP, mg/dl & $2.7 \pm 4.6$ & $1.4 \pm 1.7$ & 0.18 \\
\hline
\end{tabular}


sistance. The TREM-1 levels similarly increased in adipose tissue samples in correlation with BMI in a study with smaller sample size and without any data on the sera. The high serum TREM-1 levels in overweight patients may therefore be an early indicator of subclinical inflammation and reflect M1 macrophage overactivity in the adipose tissue. Serum TREM-1 is therefore likely to be a new easy-measurable marker that reflects subclinical inflammation in overweight patients.

An increase of TREM-1 levels has a substantial effect on inflammatory processes. Following stimulation by damage and various inflammatory stimuli, CRP can be secreted in high quantities by hepatic cells and enters the circulation within 12-48 hours. Elevated serum TREM-1 levels in overweight female participants might be associated with a subclinical low -grade inflammation related to the innate immune system, independent of the CRP levels. It is acknowledged that TREM-1 accelerates cell surface activation markers in myeloid cells, which is seen in various conditions such as: septic shock (10), obstructive sleep apnea (11), lung cancer (12), etc. Recently, Saurer et al (13) detected high serum levels of TREM-1 in inflammatory bowel disease. The substance was also over-expressed in a number of infectious diseases, such as: pneumonia and suppurated cholangitis $(14,15)$.

Interestingly, it has been revealed that TREM-1 is upregulated in atherosclerotic plaques, where destabilization triggers an inflammation. Once MMP-9 and TNF-alfa are secreted, they upregulate TREM-1 via macrophages and vascular smooth muscle in atherosclerotic symptomatic plaques. Also, MMP-9 breaks down and increases the level of membrane-linked TREM-1 (16, 17). The TREM-1 level is higher during an acute myocardial infarction and is considered a poor prognostic factor (18). We included premenopausal female participants with no atherosclerotic history aged between 18 and 45 years in our study. Thus, increasing TREM-1 look-alikes are irrelevant to atherosclerotic events. Another experiment revealed that genetic and pharmacological inhibition of TREM-1 limited atherosclerotic plaques in mice (19).

There was no difference in the fasting lipid levels between the study groups, and a relationship between TREM-1 and lipid parameters was not detected in our study.

Obesity is directly related to inflammation. Subclinical inflammation in adipose tissue and changes in adipocytokines are widely associated with an insulin resistance, type 2 diabetes mellitus, metabolic syndrome and upregulated atherosclerosis in obese individuals. Obesity-associated insulin resistance is mediated by a secretion of pro-inflammatory cytokines (TNF- $\alpha$, IL-6, and IL-1 $\beta$ ) and chemo attractants (MCP-1). These inflammatory mediators increase the chemotaxis of inflammatory cells into adipose tissues (8). Subramanian et al (20) studied the association of TREM-1 levels in tissue samples obtained by abdominal surgery with the macrophage distribution and insulin resistance. The results of their study revealed an over-expression of TREM-1 and M1 markers and down-regulation of TREM-2 and M2 markers in the omentum, subcutaneous tissue and liver biopsies of obese patients (diabetics and non-diabetics) compared to non-obese patients. Overall, the obese diabetic group yielded a significantly higher number of patients with an overexpression of M1 markers (TREM-1, CD68, CD86, CCR-7, iNOS, IFN- $\gamma$, TNF- $\alpha$, IL-6, MCP-1, CCR-2 and CCR-5) and down-regulation of M2 markers (CD206, CD163 and IL-4) in liver biopsy compared to obese non-diabetics. Obese diabetics revealed a significantly increased expression of IFN- $\gamma$ compared to obese non-diabetics that was widely associated with TREM-1 over-expression in the omentum and liver biopsy samples. The results of their study suggest a possible regulatory role of TREM-1 on the secretion of IFN- $\gamma$, and by extension, an effect on macrophage polarization and obesity-induced insulin resistance. The study population included 17 morbidly obese diabetic patients, 16 morbidly obese non-diabetic patients and 5 non-obese nondiabetic subjects. There was no overweight patient.

In another study, obese diabetics had a significantly increased TREM-1, high-mobility group box-1 (HMGB1), receptors for advanced glycation end products (RAGE), and TLR levels compared to obese non-diabetics in liver, omentum, and subcutaneous biopsies. Obesity-induced insulin resistance has also been associated with RAGE, TLR-4, and TLR-2 (21).

This study is the first to evaluate TREM-1 levels in the serum of overweight female subjects. The results of this group were significantly higher than in the subjects with a normal weight. None of the subjects included in the study was diagnosed with diabetes. However, a positive correlation between TREM-1 and BMI indicated an inflammation. The measurement of the adipose tissue levels of TREM-1 may be considered in overweight patients in future.

The study had several limitations. All the subjects were female. Serum levels of the other atherosclerotic cardiac markers and proinflammatory cytokines could not be measured. We were also unable to use techniques that are more sensitive than bioimpedance analysis (e.g. MRI, DEXA) for the analysis of body components. Further investigations on this matter are required.

In conclusion, the present study mainly showed that high serum TREM-1 levels might be an early inflammatory marker in overweight patients and therefore the overweight patients should be considered for the proinflammatory and atherosclerotic process. We found that TREM-1 might be associated with BMI in overweight patients regardless of insulin resistance. The increase in body-mass index might be related to inflammation due to adipokines independently of the antropometric measures.

\section{References}

1. Heilbronn L, Campbell L. Adipose Tissue Macrophages, Low Grade Inflammation and Insulin Resistance in Human Obesity. Curr Pharm Des 2008; 14 (12): 1225-1230.

2. Kamei N, Tobe K, Suzuki R, Ohsugi M, Watanabe T, Kubota N et al. Overexpression of Monocyte Chemoattractant Protein-1 in Adipose Tissues Causes Macrophage Recruitment and Insulin Resistance. J Biol Chem 2006; 281 (36): 26602-26614.

3. Akira S, Takeda K. Functions of toll-like receptors: lessons from KO mice. C R Biol (Internet). 2004; 327 (6): 581-589.

4. Kouassi K, Gunasekar P, Agrawal D, Jadhav G. TREM-1; Is It a Pivotal Target for Cardiovascular Diseases? J Cardiovasc Dev Dis 2018; 5 (3): 45 . 


\section{$259-262$}

5. Radaev S, Kattah M, Rostro B, Colonna M, Sun PD. Crystal Structure of the Human Myeloid Cell Activating Receptor TREM-1. Structure 2003; 11 (12): 1527-1535.

6. Kelker MS, Foss TR, Peti W, Teyton L, Kelly JW, Wüthrich K et al. Crystal Structure of Human Triggering Receptor Expressed on Myeloid Cells 1 (TREM-1) at $1.47 \AA$ A. J Mol Biol 2004; 342 (4): 1237-1248.

7. Gao S, Yi Y, Xia G, Yu C, Ye C, Tu F et al. The characteristics and pivotal roles of triggering receptor expressed on myeloid cells-1 in autoimmune diseases. Autoimmun Rev 2019; 18 (1): 25-35.

8. Subramanian S, Pallati PK, Rai V, Sharma P, Agrawal DK, Nandipati KC. Increased expression of triggering receptor expressed on myeloid cells- 1 in the population with obesity and insulin resistance. Obesity 2017; 25 (3): 527-538.

9. Ginter E, Simko V. Becoming Overweight: is there a health risk? Bratisl Lek listy 2014; 115 (9): 527-531.

10. Arízaga-Ballesteros V, Alcorta-García MR, Lázaro-Martínez LC, Amézquita-Gómez JM, Alanís-Cajero JM, Villela L et al. Can sTREM-1 predict septic shock \&amp; death in late-onset neonatal sepsis? A pilot study. Int J Infect Dis 2015; 30: 27-32.

11. Kim J, Gozal D, Bhattacharjee R, Kheirandish-Gozal L. TREM1 and Pentraxin-3 Plasma Levels and Their Association with Obstructive Sleep Apnea, Obesity, and Endothelial Function in Children. Sleep 2013; 36 (6): 923-931.

12. Ho CC, Liao WY, Wang CY, Lu YH, Huang HY, Chen HY et al. TREM-1 Expression in Tumor-associated Macrophages and Clinical Outcome in Lung Cancer. Am J Respir Crit Care Med 2008; 177 (7): 763-770.

13. Saurer L, Rihs S, Birrer M, Saxer-Seculic N, Radsak M, Mueller C et al. Elevated levels of serum-soluble triggering receptor expressed on myeloid cells- 1 in patients with IBD do not correlate with intestinal TREM-1 mRNA expression and endoscopic disease activity. J Crohn's Colitis 2012; 6 (9): 913-923.
14. Miao Y, Liu Z-J, Gong J-P, Wei S-D, Xu F-L, Chen Z-Z. (Expression of human triggering receptor expressed on myeloid cells 1 in peripheral blood mononuclear cells of patients with acute obstructive suppurative cholangitis). Nan Fang Yi Ke Da Xue Xue Bao 2009; 29 (11): 2179-2181.

15. Hommes TJ, Hoogendijk AJ, Dessing MC, van't Veer C, Florquin S, Colonna M et al. Triggering receptor expressed on myeloid cells-1 (TREM-1) improves host defence in pneumococcal pneumonia. J Pathol 2014; 233 (4): 357-367.

16. Rao VH, Rai V, Stoupa S, Subramanian S, Agrawal DK. Tumor necrosis factor- $\alpha$ regulates triggering receptor expressed on myeloid cells1-dependent matrix metalloproteinases in the carotid plaques of symptomatic patients with carotid stenosis. Atherosclerosis 2016; 248: 160-169.

17. Zysset D, Weber B, Rihs S, Brasseit J, Freigang S, Riether C et al. TREM-1 links dyslipidemia to inflammation and lipid deposition in atherosclerosis. Nat Commun 2016; 7: 13151.

18. Wang YK, Tang JN, Shen YL, Hu B, Zhang CY, Li MH et al. Prognostic Utility of Soluble TREM-1 in Predicting Mortality and Cardiovascular Events in Patients With Acute Myocardial Infarction. J Am Heart Assoc 2019; 7 (12) pii: e008985.

19. Joffre J, Potteaux S, Zeboudj L, Loyer X, Boufenzer A, Laurans L et al. Genetic and Pharmacological Inhibition of TREM-1 Limits the Development of Experimental Atherosclerosis. J Am Coll Cardiol 2016; 68 (25): 2776-2793.

20. Subramanian S, Pallati PK, Sharma P, Agrawal DK, Nandipati KC. TREM-1 associated macrophage polarization plays a significant role in inducing insulin resistance in obese population. J Transl Med 2017; 15 (1): 1-18.

21. Subramanian S, Pallati PK, Sharma P, Agrawal DK, Nandipati KC. Significant association of TREM-1 with HMGB1, TLRs and RAGE in the pathogenesis of insulin resistance in obese diabetic populations. Am J Transl Res 2017; 9 (7): 3224-3244. 